\title{
Research of Dosimetry Parameters in Small Electron Beams
}

\author{
Nazmiye Donmez Kesen, Aydin Cakir, Murat Okutan, and Hatice Bilge \\ Radiotherapy Department, Istanbul University Oncology Institute, Fatih, Capa, 34390 Istanbul, Turkey \\ Correspondence should be addressed to Nazmiye Donmez Kesen; nazo94@gmail.com
}

Received 29 November 2013; Accepted 17 February 2014; Published 25 March 2014

Academic Editor: Jakrapong Kaewkhao

Copyright (C) 2014 Nazmiye Donmez Kesen et al. This is an open access article distributed under the Creative Commons Attribution License, which permits unrestricted use, distribution, and reproduction in any medium, provided the original work is properly cited.

\begin{abstract}
In this study, dose distributions and outputs of circular fields with dimensions of $5 \mathrm{~cm}$ and smaller, for 6 and $9 \mathrm{MeV}$ nominal energies from the Siemens ONCOR Linac, were measured and compared with data from a treatment planning system using the pencil beam algorithm in electron beam calculations. All dose distribution measurements were performed using the GafChromic EBT film; these measurements were compared with data that were obtained from the Computerized Medical Systems (CMS) XiO treatment planning system (TPS). Output measurements were performed using GafChromic EBT film, an Advanced Markus ion chamber, and thermoluminescent dosimetry (TLD). Although it is used in many clinics, there is not a substantial amount of detailed information in the literature about use of the pencil beam algorithm to model electron beams. Output factors were consistent; differences from the values obtained from the TPS were at maximum. When the dose distributions from the TPS were compared with the measurements from the ion chamber and GafChromic EBT films, it was observed that the results were consistent with $2 \mathrm{~cm}$ diameter fields and larger, but the outputs for $1 \mathrm{~cm}$ diameter fields and smaller were not consistent.
\end{abstract}

\section{Introduction}

In recent years, electron beams, rather than low X-ray beams, have been used for the treatment of superficial lesions, because of the different dosage characteristics of the two sources. Tumours which are close to critical body structures require small directionality error margins and thus require small treatment fields. Electron beams have uniform dose distribution at the surface and a rapid decrease in the dose below a specific depth [1].

Electron beam therapy is an indispensable practice in radiotherapy, and therefore most commercial treatment planning systems use electron beam programs alongside photon planning programs. Electron beam dose calculations were originally based on empirical functions that utilised ray line geometries [2,3]. More advanced pencil beam algorithms are based on multiple scattering theories [4, 5]. The major limitation of both empirical methods and pencil beam algorithms is their inability to predict depth dose distributions and accurately monitor units for small field sizes. A Monte Carlo-based dose calculation algorithm has been investigated by several groups. The accuracy of implementation of this algorithm was found to be more reliable than that of other algorithms. A commercial Monte
Carlo-based dose calculation algorithm has become available for electron beam treatment planning systems. Nevertheless, pencil beam algorithms are still used for electron beam treatment planning in many oncology clinics.

There are several theoretical and experimental methods described in the literature for determination of the dose distribution and prediction of the electron beam output factors. Film dosimetry is the most rapid method to obtain the dose distribution and related output factors.

Dose distributions for small circular electron beams from the Siemens Oncor linear accelerator using GafChromic EBT films were measured. Output factors were measured using GafChromic EBT films, TLD, and the Advanced Markus ion chamber. The measured values were compared with CMS $\mathrm{XiO}$ treatment planning system data, which uses the pencil beam algorithm.

\section{Methods and Materials}

2.1. Isodose Distribution Measurements. The source of electron beams used for this study was a high energy linear accelerator (Siemens Oncor, Germany). The linear accelerator had a circular cone with diameter $5 \mathrm{~cm}$ that was used as 
the standard cone. Cut-outs were prepared to achieve fields with diameters of $1,2,3$, and $4 \mathrm{~cm}$.

Dose distribution measurements were made for these circular fields, $1-5 \mathrm{~cm}$ in diameter, using a MP3-M automatic water phantom and a Semiflex $(0.125 \mathrm{cc})$ ion chamber (PTWNew York Corp., Hicksville, NY), with a source-to-surface distance (SSD) of $100 \mathrm{~cm} .1,2,3$, and $4 \mathrm{~cm}$ cut-outs were placed into the $5 \mathrm{~cm}$ diameter standard circular cone. For profile measurements range reference $\left(R_{\text {ref }}\right)$, dose range of $85 \%\left(R_{85}\right)$, dose range of $(85 \%) / 2\left(R_{85 / 2}\right)$, dose range of $50 \%\left(R_{50}\right)$, and practical range $\left(R_{p}\right)$ depth values were used for all electron energies and were obtained from percentage depth dose curves measured using a $10 \times 10 \mathrm{~cm}^{2}$ standard cone. Depth dose measurements were repeated for all small fields. Profiles and depth dose curves were put into the PTW Verisoft software program (PTW-New York Corp., Hicksville, $\mathrm{NY}$ ) and isodose curves were obtained.

In film dosimetry, GafChromic EBT films (International Specialty Products, Wayne, NJ, USA) were used because of their characteristic null energy response. GafChromic EBT films are more sensitive than older radiochromic films and are readily available for photon dosimetry as well as electron dosimetry [6]. The measurable dose range is $1-800 \mathrm{cGy}$. EBT films have a relatively small effective thickness; as such the effective measurement point was neglected. For calibration of films, a $9 \mathrm{MeV}$ electron beam was used; each film was oriented perpendicular to the central axis of the electron beam, at the centre of the beam, in a solid water phantom. Films were placed at the depth of maximum dose $(20 \mathrm{~mm})$. The calibration curve was obtained by exposing films in a $10 \times 10 \mathrm{~cm}^{2}$ field, with doses ranging from 10 to $800 \mathrm{cGy}$. All exposed films were considered after $24 \mathrm{~h}$, because the darkening saturation of films must be allowed to occur [7].

Films were scanned using a flatbed colour scanner (EPSON America Inc., Long Beach, CA), collected using its software program, and analyzed using the ImageJ program; films were separated into three colours: red, blue, and green. For optimum contrast, the red image was selected for film dosimetry, because the peak absorption of the EBT film is in the red region of the visible spectrum. Besides measuring the characteristics of all exposed films, a nonexposed film was scanned to measure the background effect. All films were placed inside the scanner in the same direction, because of the orientation problem. Permeability at the centre of the exposed films was observed using the Mephysto mcc software program (PTW-New York Corp., Hicksville, NY) and a calibration curve was generated.

To obtain the isodose distributions, films were oriented parallel to the electron beam in a solid water phantom. The setup is shown in Figure 1. Alongside the $5 \mathrm{~cm}$ standard circular cone, the 1-4 cm diameter cut-outs were used, and films were exposed with 6 and $9 \mathrm{MeV}$ nominal energies. Isodose curves were generated using the calibration curve in the PTW Verisoft program.

The XiO therapy planning system (TPS) (CMS-Elekta $A B$, Stockholm), with its pencil beam algorithm, was used for calculation of the isodose curves. XiO TPS does not support circular cones; the system only has square field electron beam

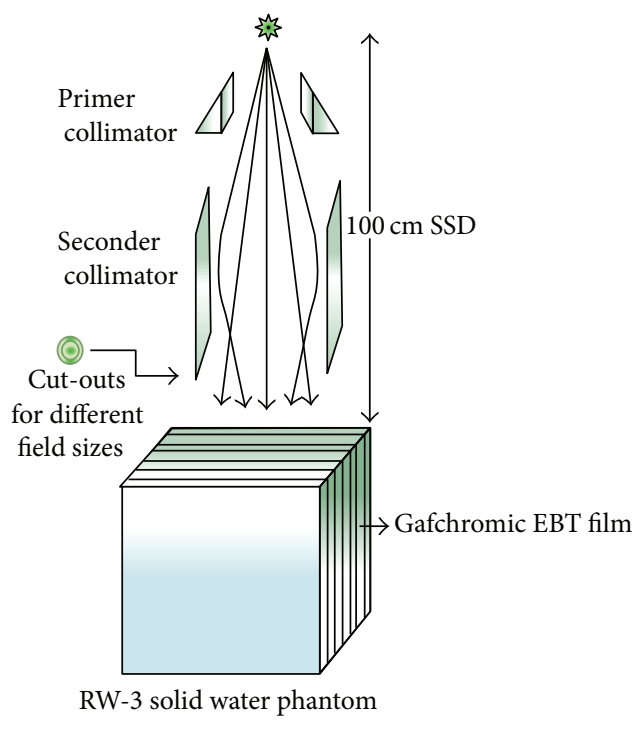

FIGURE 1: Solid water phantom setup used for isodose distribution measurements. Gafchromic films were aligned parallel to the electron beam in solid water phantom.

data. Therefore, the smallest cone, $10 \times 10 \mathrm{~cm}^{2}$, was blocked to obtain $5,4,3,2$, and $1 \mathrm{~cm}$ field sizes in the planning system. Isodose distributions were calculated using the pencil beam algorithm and fluence maps of isodoses were obtained for all energies using a solid phantom. Fluence maps were transferred into the PTW Verisoft program and converted into isodose curves.

To provide comparisons of multidimensional dose distributions, dose comparison tools such as gamma dose distribution, distance-to-agreement (DTA), and dose difference (DD) have been developed $[8,9]$. The gamma dose distribution tool was used in our study. A DD of $3 \%$ and $3 \mathrm{~mm}$ DTA gamma evaluation parameters were used for analysis. Sagittal isodose distributions along the central axis were measured using EBT film and were compared with data that were calculated using the treatment planning system.

2.2. Output Factor Measurements. Output factor measurements were performed for 6 and $9 \mathrm{MeV}$ electron beams in a $40 \times 40 \mathrm{~cm}^{2}$ solid water phantom at $100 \mathrm{~cm}$ SSD, using the PTW Unidos (Freiburg, Germany) electrometer, Advanced Markus ion chamber, films, and TLD. Reference depths were determined for each electron energy from the IAEA 398 protocol, using the formula below: [10]

$$
D_{\text {ref }}=0.6 R_{50}-0.1 \mathrm{~g} / \mathrm{cm}^{2} \quad\left(R_{50}, \mathrm{~g} / \mathrm{cm}^{2}\right),
$$

where $R_{50}\left(\mathrm{~cm}\right.$ or $\left.\mathrm{g} / \mathrm{cm}^{2}\right)$ is the depth of the beam when the dose is equal to $50 \%$ of the maximum dose.

The $D_{\text {ref }}$ values for 6 and $9 \mathrm{MeV}$ electron beams were 1.3 and $2 \mathrm{~cm}$, respectively. Outputs were measured for 5,4 , 3,2 , and $1 \mathrm{~cm}$ circular fields using the cerrobend cut-outs within a $5 \mathrm{~cm}$ circular cone. Output factors were obtained and normalised to the $10 \times 10 \mathrm{~cm}^{2}$ field. 


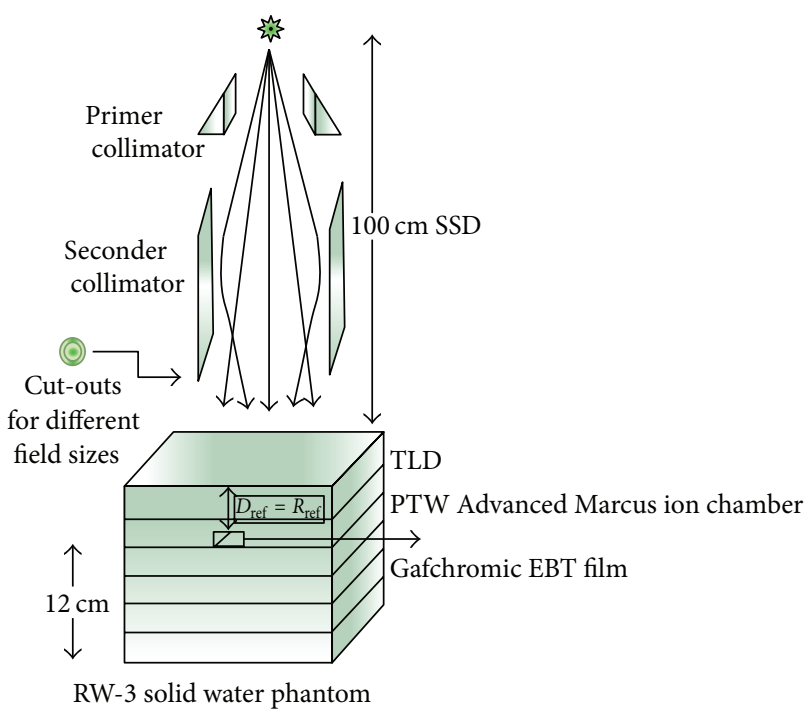

Figure 2: Output factor measurements were obtained with solid water phantoms which were aligned perpendicular to the electron beam.

Output factors were measured using the Advanced Markus ion chamber (PTW-New York Corp., Hicksville, NY), GafChromic EBT film, and TLD. Phantoms were positioned perpendicular to the beam direction. $100 \mathrm{MU}$ was defined for both the ion chamber and TLD measurements. The experimental setup is shown in Figure 2.

GafChromic EBT film was oriented perpendicular to the direction of the incident electron beam, and care was taken to align the beam to the centre of the film. For all field sizes, $400 \mathrm{MU}$ was considered to provide sufficient darkness on the films. All films were scanned the following day and separated into colour scales; the red measurement was selected and recorded. Point permeability, which was measured at the field centre, was converted into a dose (Gy). All films used were from the same batch and irradiated on the same day.

For TLD measurements, $1 \times 1 \times 1 \mathrm{~mm}^{3}$ TLD rods and a GR-200R1 Fimel reader were used. Ternary groups were not used because of the small measurement size considered. One TLD was positioned on the custom-built phantom and irradiated with $100 \mathrm{MU}$ at the reference depth. Irradiation was repeated five times and the average of the readings was taken. TLD rods were read with the Fimel-LTM; output factors were obtained and normalised to a $10 \times 10 \mathrm{~cm}^{2}$ field size.

In the CMS TPS, a $10 \times 10 \mathrm{~cm}^{2}$ standard cone was blocked to generate $5,4,3,2$, and $1 \mathrm{~cm}$ diameter circular fields. For 6 and $9 \mathrm{MeV}$ electron beams, the MU calculation was carried out in the TPS, considering $100 \mathrm{cGy}$ at the reference depth along the central axis. Output factors were determined from the MU values. Output factors obtained from the TPS were compared with the factors that were found using other dosimetric methods.

\section{Results and Discussion}

For 6 and $9 \mathrm{MeV}$ electron beams, $100 \%\left(d_{\max }\right), 90 \%, 80 \%$, $50 \%, R_{p}, E_{p 0}$, and $E_{0}$ values were obtained from the depth

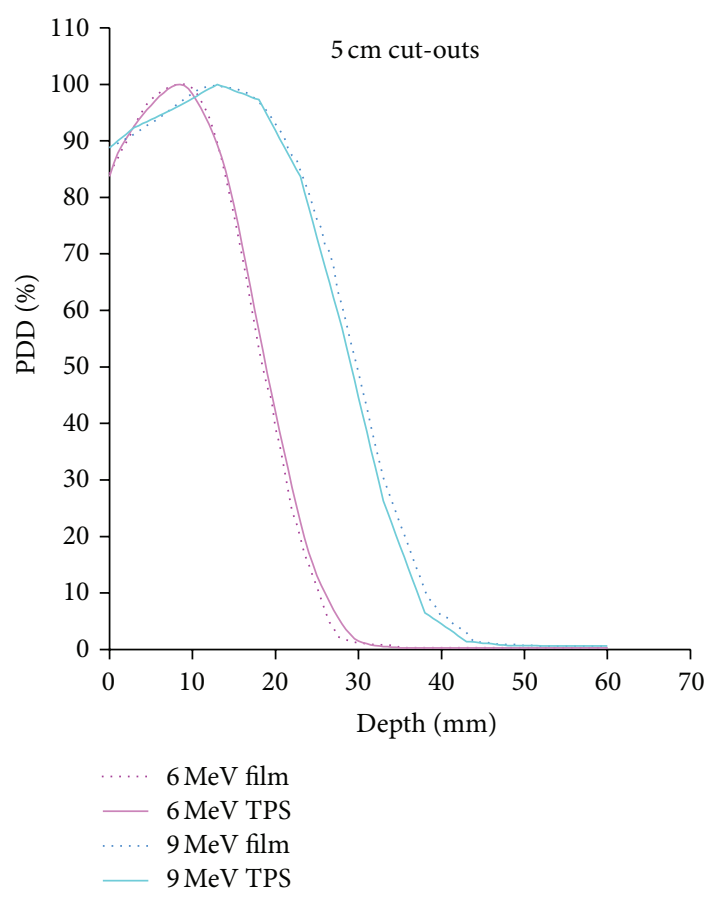

Figure 3: Percent depth dose curve comparisons between measured data using Semiflex ion chamber and EBT films using $5 \mathrm{~cm}$ circular cone at $100 \mathrm{~cm}$ SSD. All curves are normalized to $100 \%$. As measurements made by Semiflex ion chamber are represented by solid lines, EBT film measurements are represented by dotted lines.

dose measurements. These were carried out using a Semiflex ion chamber with a volume of $0.125 \mathrm{cc}$, and the $10 \times 10 \mathrm{~cm}^{2}$ field values are shown in Table 1.

For the $6 \mathrm{MeV}$ electron beam, the $d_{\max }$ value obtained from the Semiflex ion chamber $(0.125 \mathrm{cc})$ is $12 \mathrm{~mm}$ in the $10 \times 10 \mathrm{~cm}^{2}$ square field size. The depth dose characteristics of electrons change dramatically with decreasing field sizes. For all electron energies, the maximum depth decreases with decreasing field sizes. In other words, when the field size was reduced, the maximum dose point moved closer to the surface. The most probable reason for this is the chamber volume effect. For chambers, therapeutic ranges decrease with field size, and the falloff region of the curve becomes less steep. This is consistent with Sharma et al's observations [11]. In addition, with decreasing field size, a decreasing degree of lateral electronic equilibrium will be present at the central axis, and the depth dose and output factors will show large sensitivity to the field shape and size [1].

Figure 3 shows a comparison of the measured data that were obtained from the Semiflex ion chamber and EBT films for a $5 \mathrm{~cm}$ circular cone at $100 \mathrm{~cm}$ SSD. The agreement between calculated and measured data is good.

The depth dose measurements obtained from the Semiflex ion chamber and film were converted to isodoses using the PTW Verisoft program. These isodoses were compared with isodoses generated from TPS. Comparisons were carried out using the gamma index method. For this method, the tolerance limit selected was $3 \mathrm{~mm}$ DTA, 3\% DD. When the dose distributions from the TPS were compared with 
TABLE 1: $100 \%\left(d_{\max }\right), 90 \%, 80 \%, 50 \%, R_{p}, E_{p 0}$, and $E_{0}$ values obtained from the depth dose measurements for 6 and $9 \mathrm{MeV}$ electron beams.

\begin{tabular}{lcccccccc}
\hline & $10 \times 10 \mathrm{~cm}^{2}$ & $R_{100}(\mathrm{~mm})$ & $R_{90}(\mathrm{~mm})$ & $R_{80}(\mathrm{~mm})$ & $R_{50}(\mathrm{~mm})$ & $R_{p}(\mathrm{~mm})$ & $E_{p 0}(\mathrm{~mm})$ & $E_{0}(\mathrm{~mm})$ \\
\hline $6 \mathrm{MeV}$ & Semiflex ion chamber & 12 & 16.77 & 18.59 & 22.68 & 28.80 & 5.94 & 5.29 \\
$9 \mathrm{MeV}$ & Semiflex ion chamber & 19.50 & 27.66 & 30.54 & 36.41 & 45.52 & 9.28 & 8.55 \\
\hline
\end{tabular}

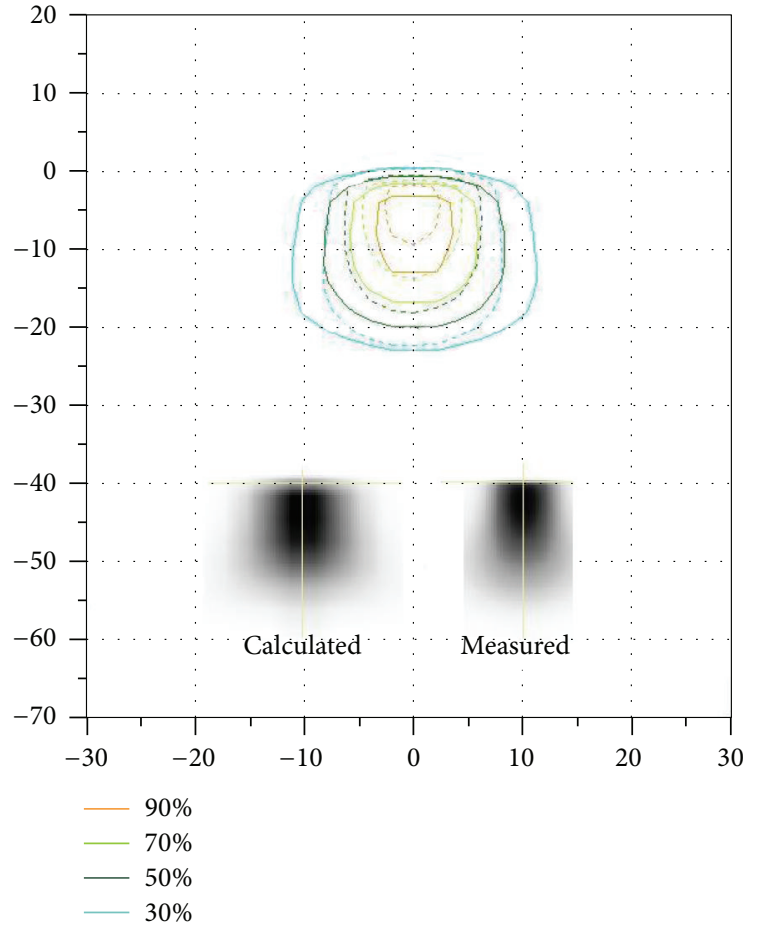

(a)

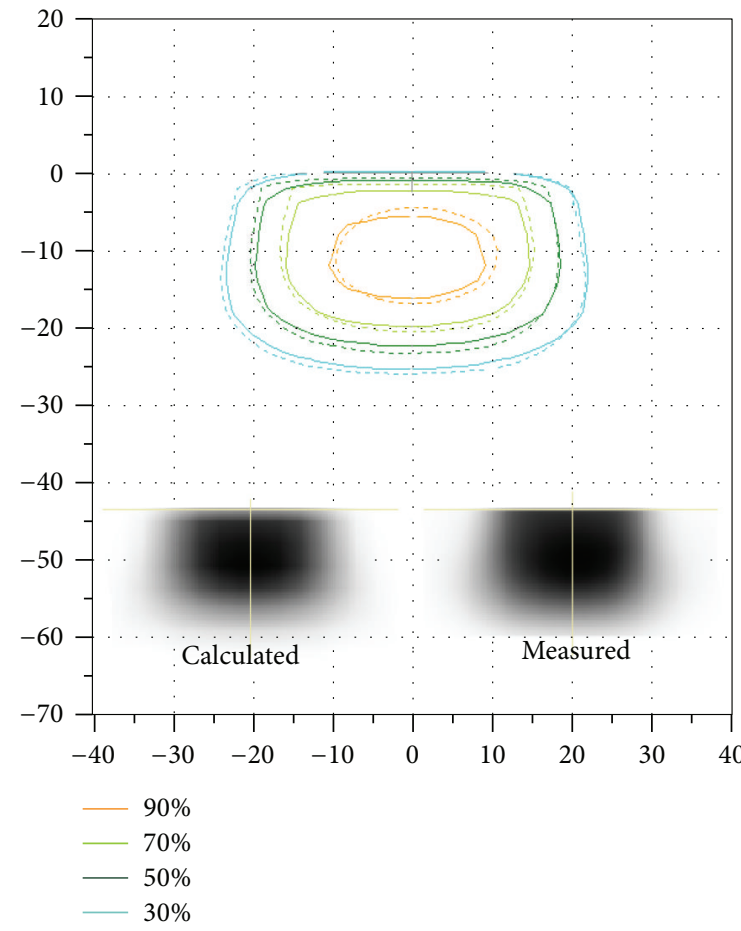

(b)

FIGURE 4: Sagittal isodose comparisons of a $6 \mathrm{MeV}$ beam along the central axis for 1 (a) $\mathrm{cm}$ and 4 (b) $\mathrm{cm}$ circular cutouts using a $5 \mathrm{~cm}$ diameter circular cone. Solid lines represent calculations and dotted lines represent measurements ( $x$-axis and $y$-axis are in $\mathrm{cm}$ ).

those from the Semiflex ion chamber and EBT films, it was seen that the results were consistent with field sizes of $2 \mathrm{~cm}$ diameter and larger. For $1 \mathrm{~cm}$ diameter fields, isodoses were incompatible at the central axis for 6 and $9 \mathrm{MeV}$ electron beams. With increasing energy and decreasing field size, the isodoses of the individual methods are no longer consistent with each other.

Figures 4 and 5 show, as an example, a sagittal isodose comparison between calculated and measured data for 6 and $9 \mathrm{MeV}$ beams, at $100 \mathrm{~cm}$ SSD using $4 \mathrm{~cm}$ and $1 \mathrm{~cm}$ cut-out sizes.

Outputs obtained using different measurement methods for 6 and $9 \mathrm{MeV}$ nominal energies, for field sizes of 5, 4, 3, 2, and $1 \mathrm{~cm}$ diameter, are shown in Table 2 . All measurements were made with a cone distance of $95 \mathrm{~cm}$.

TLD results have average values for all fields between the values obtained for ion chamber and film measurements. However, generally, with increasing energy level and field size, output factor differences decrease.

For all energies and field sizes, output factors were consistent; differences were found to be, at maximum, $27 \%$, compared with the values from the TPS.
In the literature, there is no evidence of studies concerning electron beam outputs for field sizes smaller than $5 \times$ $5 \mathrm{~cm}^{2}$ using the pencil beam algorithm. In recent years, it has been discovered that the Monte Carlo algorithm gives more accurate results than the pencil beam algorithm.

The results of comparisons using gamma analysis (3\% and $3 \mathrm{~mm}$ ) are shown in Figure 6. The agreement between measured and calculated dose distribution values was very good for both 4 and $3 \mathrm{~cm}$ cut-outs. For the $2 \mathrm{~cm}$ cut-out, agreement was good at the centre of the field but poor at the edge. Agreement was very poor for the $1 \mathrm{~cm}$ cut-out at both 6 and $9 \mathrm{MeV}$.

It was observed that results obtained at $6 \mathrm{MeV}$ were consistent, and consistency was good at both the high and low dose regions at this energy. However, with increasing energy, this consistency reduced. With increasing energy, comparable results were obtained in the low dose region but not in the high dose region. This is due to the high dose falling region; the accuracy of the gamma index comparison decreases.

A comparison was made between the calculated and measured depth doses, isodose distributions, and outputs for 6 and $9 \mathrm{MeV}$ and $4,3,2$, and $1 \mathrm{~cm}$ circular cut-outs using 
TABle 2: Percentage differences between calculated and measured outputs from different measurement methods at 6 and $9 \mathrm{MeV}$ nominal energies for 5, 4, 3, 2, and $1 \mathrm{~cm}$ field sizes. For both energies, $10 \times 10 \mathrm{~cm}^{2}$ field size was assumed for TPS values.

\begin{tabular}{|c|c|c|c|c|c|}
\hline & $5 \mathrm{~cm}$ & $4 \mathrm{~cm}$ & $3 \mathrm{~cm}$ & $2 \mathrm{~cm}$ & $1 \mathrm{~cm}$ \\
\hline \multicolumn{6}{|l|}{$6 \mathrm{MeV}$} \\
\hline Advanced Markus-TPS & $21 \%$ & $24 \%$ & $27 \%$ & $21 \%$ & $17 \%$ \\
\hline EBT film-TPS & $18 \%$ & $20 \%$ & $21 \%$ & $15 \%$ & $3 \%$ \\
\hline TLD-TPS & $20 \%$ & $22 \%$ & $27 \%$ & $15 \%$ & $9 \%$ \\
\hline \multicolumn{6}{|l|}{$9 \mathrm{MeV}$} \\
\hline Advanced Markus-TPS & $10 \%$ & $14 \%$ & $19 \%$ & $17 \%$ & $16 \%$ \\
\hline EBT film-TPS & $7 \%$ & $10 \%$ & $13 \%$ & $13 \%$ & $7 \%$ \\
\hline TLD-TPS & $8 \%$ & $10 \%$ & $15 \%$ & $12 \%$ & $5 \%$ \\
\hline
\end{tabular}

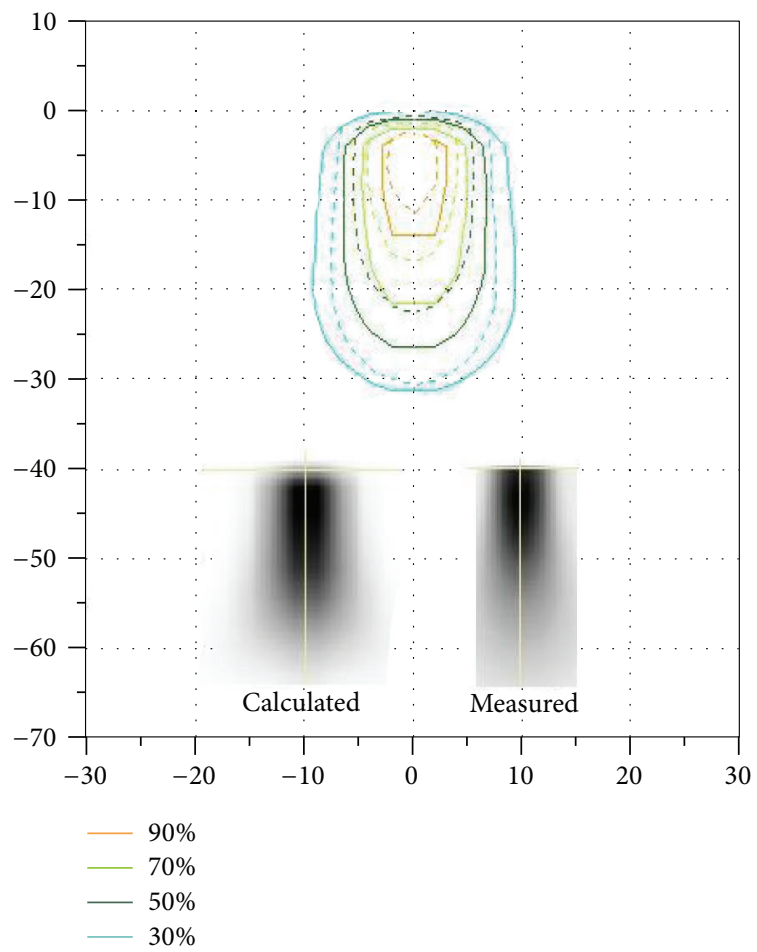

(a)

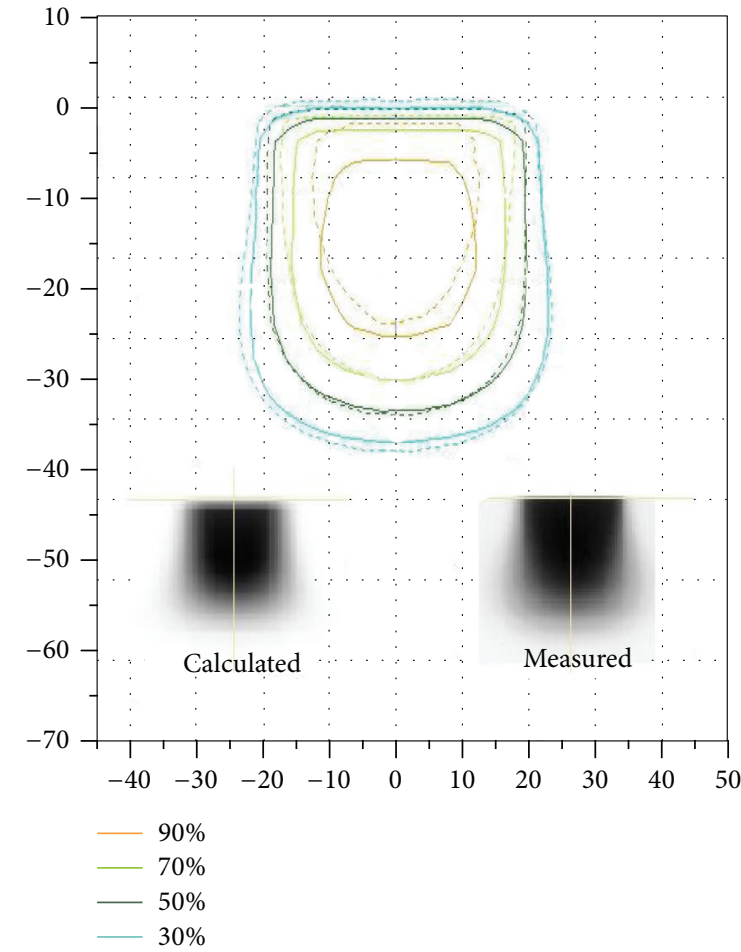

(b)

FIGURE 5: Sagittal isodose comparisons of a $9 \mathrm{MeV}$ beam along the central axis for 1 (a) $\mathrm{cm}$ and 4 (b) $\mathrm{cm}$ circular cut-outs using a $5 \mathrm{~cm}$ diameter circular cone. Solid lines represent calculations and dotted lines represent measurements ( $x$-axis and $y$-axis are in $\mathrm{cm})$.

a $5 \mathrm{~cm}$ standard circular cone. Our study shows that pencil beam algorithm can be used to accurately predict depth doses and isodose distributions for fields with diameter as small as $3 \mathrm{~cm}$ at $100 \mathrm{~cm}$ SSD. When the cutout size is smaller than $3 \mathrm{~cm}$, the circular calculated dose distribution can differ significantly from the measurement at $100 \mathrm{~cm}$ SSD. MUs obtained from the planning system were different from the measurements for all circular cutouts using the $5 \mathrm{~cm}$ circular cone. This is consistent with results reported by Xu et al. [12]. The most important limitation of the pencil beam algorithm is its failure to predict depth dose distributions and accurate MUs for field sizes smaller than the extent of the lateral scatter equilibrium [12]. It has also been observed in our study that pencil beam algorithm should not be used for MU calculations for small electron field sizes.

The American Association of Physics in Medicine (AAPM) recommends that because of their high spatial resolution, films should be primarily chosen for electron beam dosimetry. This is supported by the fact that the film dose-response curve was found to be weakly dependent on the electron beam energy (within $\pm 4 \%$ ) [13]. In addition, in our output factor measurements, it was seen that GafChromic EBT film measurement results were closer to the calculated results than other dosimetric methods. In the literature, it is suggested that before using detectors for determination of the output factors of small electron fields, $d_{\max }$ values should be observed by film dosimetry [11].

\section{Conclusion}

The dosimetry of small electron fields is highly critical. Output measurements of electron fields smaller than the extent of the lateral scatter equilibrium must be carefully made using appropriate dosimetry. Furthermore, for the 


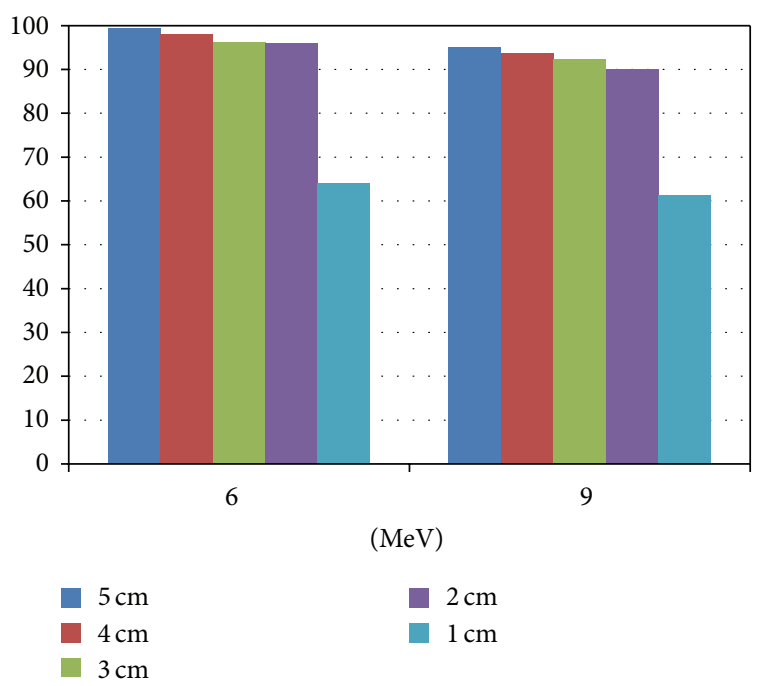

Figure 6: Gamma analysis results for beams with 6 and $9 \mathrm{MeV}$ energy, for cut-out diameters of $4,3,2$, and $1 \mathrm{~cm}$ using a $5 \mathrm{~cm}$ circular cone at $100 \mathrm{~cm} \mathrm{SSD} \mathrm{(} y$-axis is \%).

patient-time calculation, output factors that were obtained by the most appropriate dosimetric method should be used, not the values from the TPS that uses the pencil beam algorithm.

At the conclusion of this study, it was observed that in the CMS XiO TPS, calculated using the pencil beam algorithm, the dose distributions of electron treatment fields that were created with circular cut-outs of 4,3 , and $2 \mathrm{~cm}$ diameter were appropriate for patient treatment, but those created using a $1 \mathrm{~cm}$ diameter field were not. Additionally, the pencil beam algorithm is not convenient for MU calculations in electron dosimetry.

\section{Conflict of Interests}

The authors declare that there is no conflict of interests regarding the publication of this paper.

\section{References}

[1] E. B. Podgorsak, Radiation Oncology Physics: a Handbook for Teachers and Students, International Atomic Energy Agency, Vienna, Austria, 2005.

[2] J. Cassidy, D. Bisset, and R. Obe, Oxford Handbook of Oncology, Oxford University Press, New York, NY, USA, 2002.

[3] E. Sternick, "Algorithms for computerized treatment planning," in Practical Aspects of Electron Beam Treatment Planning: Proceedings of the Practical Aspects of Electron Beam Treatment Planning Symposium, July 31, 1977, Cincinnati, Ohio, New York, C. G. Orton and F. Baagne, Eds., p. 52, American Institute of Physics, 1978.

[4] S. C. Sharma, D. L. Wilson, and B. Jose, "Dosimetry of small fields for Therac 20 electron beams," Medical Physics, vol. 11, no. 5, pp. 697-702, 1984.

[5] K. R. . Hogstrom, G. Starkschall, and A. S. Shiu, "Dose calculation algorithms for electron beams," in Advances in Radiation Oncology Physics: Dosimetry, Treatment Planning and
Brachytherapy. American Institute of Physics Monograph, J. A. Purdy, Ed., p. 900, American Institute of Physics, New York, NY, USA, 1992.

[6] H. Bilge, N. Ozbek, M. Okutan, A. Cakir, and H. Acar, "Surface dose and build-up region measurements with wedge filters for 6 and $18 \mathrm{MV}$ photon beams," Japanese Journal of Radiology, vol. 28, no. 2, pp. 110-116, 2010.

[7] ISP International Speciality Products, "Gafchromic EBT SelfDeveloping Film for Radiotherapy Dosimetry," 2007, http:// onlinel.ispcorp.com/_layouts/Gafchromic/content/products/ ebt/pdfs/EBTwhitepaper.pdf.

[8] D. A. Low, W. B. Harms, S. Mutic, and J. A. Purdy, "A technique for the quantitative evaluation of dose distributions," Medical Physics, vol. 25, no. 5, pp. 656-661, 1998.

[9] D. A. Low and J. F. Dempsey, "Evaluation of the gamma dose distribution comparison method," Medical Physics, vol. 30, no. 9, pp. 2455-2464, 2003.

[10] IAEA, "Absorbed dose determination in external beam radiotherapy: an international code of practice for dosimetry based on standarts of absorbed dose to water," Tech. Rep. 398, International Atomic Energy Agency, 2000.

[11] S. C. Sharma, M. W. Johnson, and M. S. Gossman, "Practical considerations for electron beam small field size dosimetry," Medical Dosimetry, vol. 30, no. 2, pp. 104-106, 2005.

[12] Z. Xu, S. E. Walsh, T. P. Telivala, A. G. Meek, and G. Yang, "Evaluation of the eclipse electron Monte Carlo dose calculation for small fields," Journal of Applied Clinical Medical Physics, vol. 10, no. 3, pp. 75-85, 2009.

[13] F.-C. Su, Y. Liu, S. Stathakis, C. Shi, C. Esquivel, and N. Papanikolaou, "Dosimetry characteristics of Gafchromic EBT film responding to therapeutic electron beams," Applied Radiation and Isotopes, vol. 65, no. 10, pp. 1187-1192, 2007. 


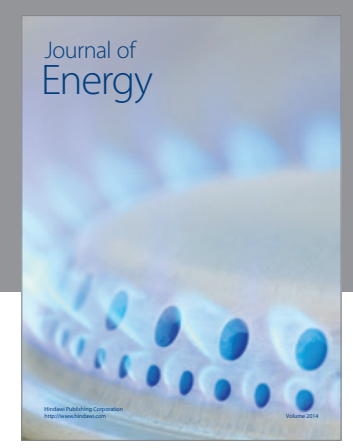

Journal of

Industrial Engineering
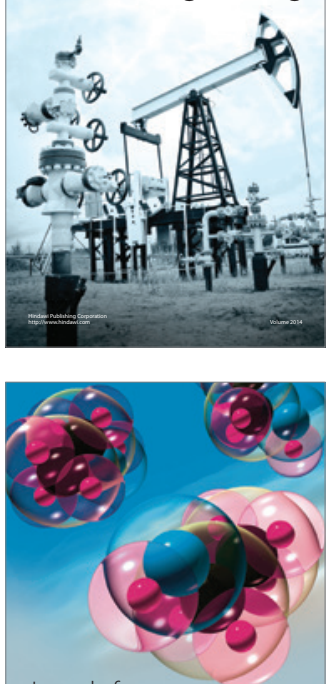

Fuels
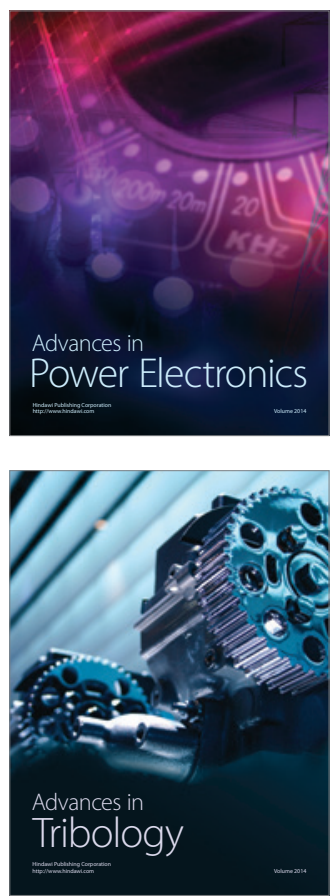

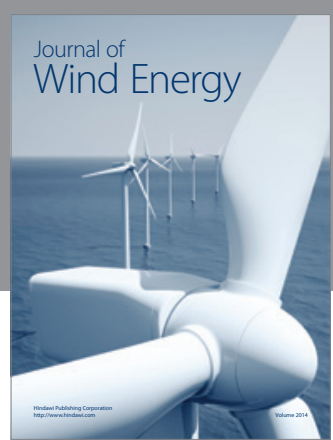

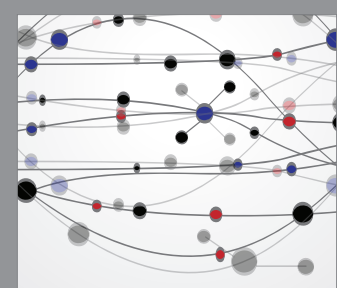

The Scientific World Journal

Submit your manuscripts at http://www.hindawi.com

Journal of

Structures
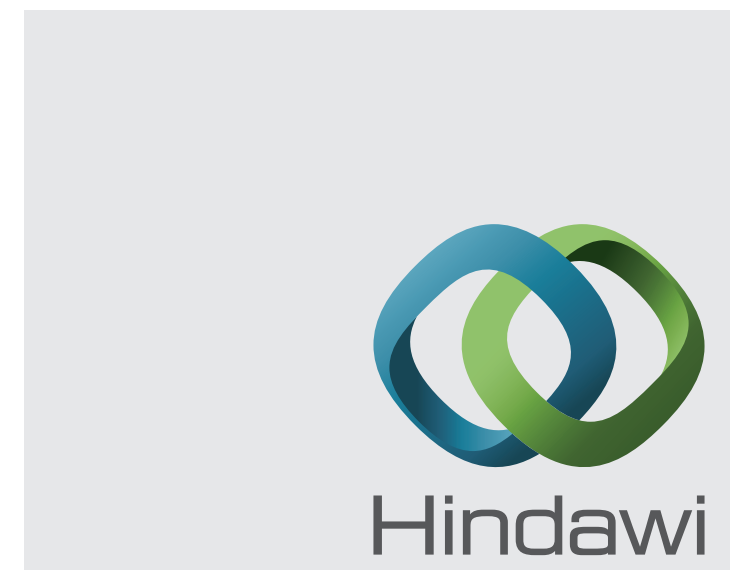

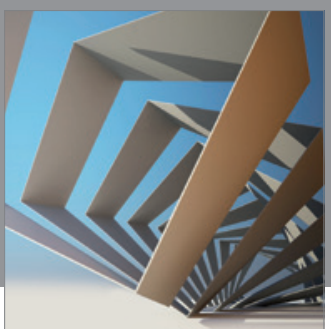

Rotating

Machinery
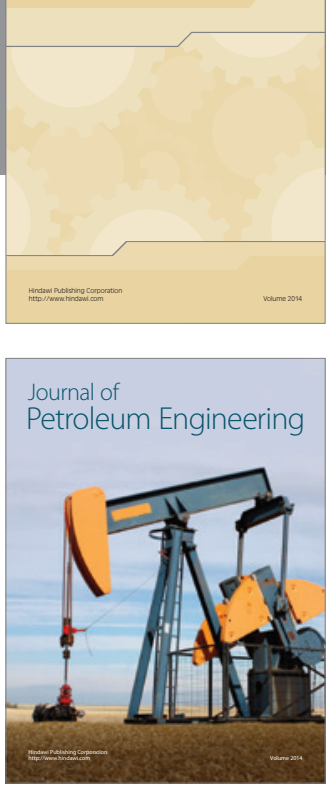

Journal of

Solar Energy
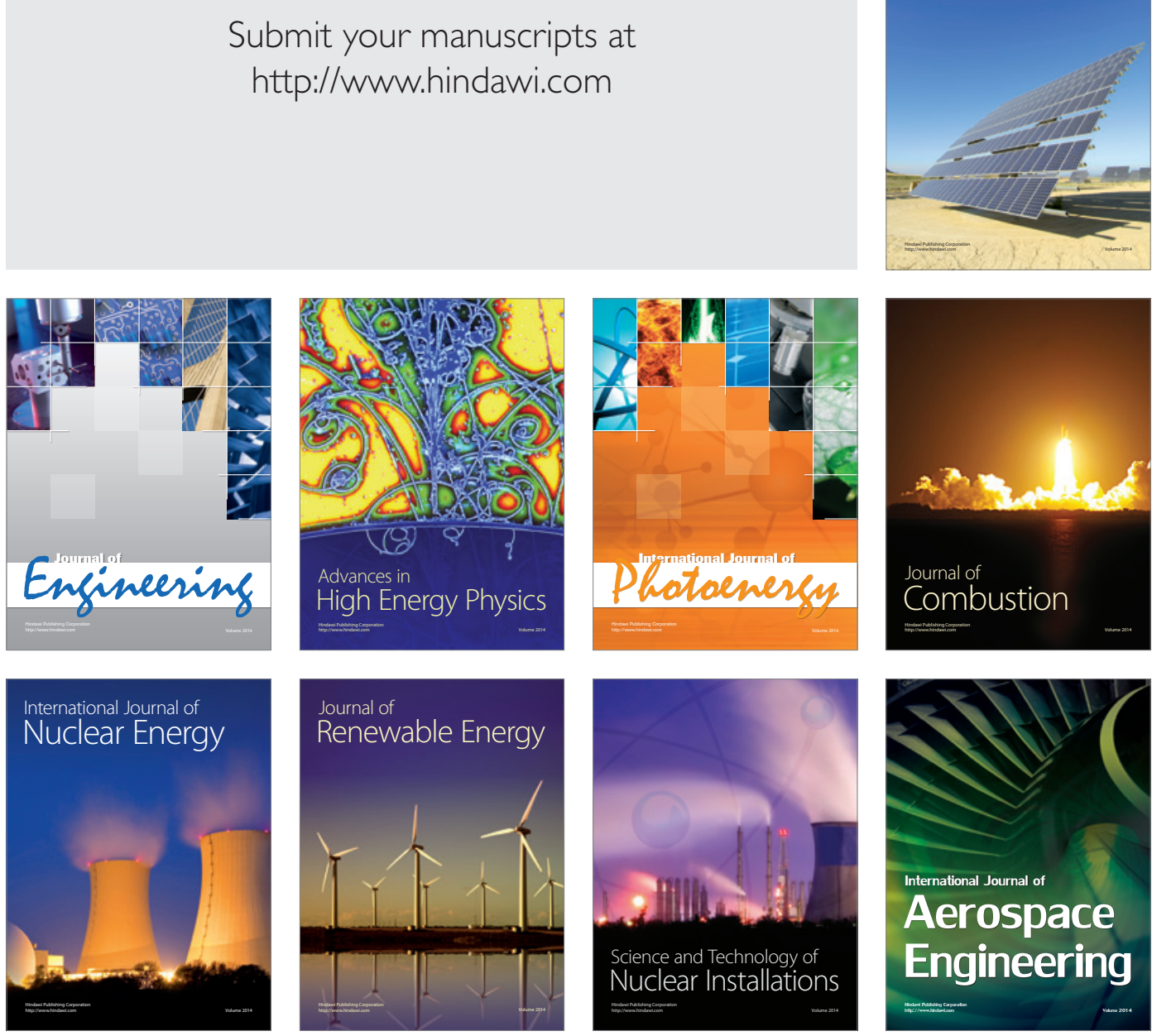